\title{
A comparison of machine-learning techniques for the prediction of the auroral electrojet index
}

\author{
Sumesh Gopinath and P R Prince*®o \\ Department of Physics, University College, Trivandrum, Kerala 695 034, India. \\ *Corresponding author.e-mail: princerprasad@gmail.com
}

MS received 18 November 2017; revised 4 December 2018; accepted 11 February 2019; published online 19 June 2019

The modern 'machine-learning models' are a section of artificially intelligent machines used to implement complex models, which can learn and improve from experience with respect to certain class of jobs, without being specifically programmed. In the present analysis, a comparative study is made of the popular machine-learning techniques regarding the prediction of auroral activity as reflected by the auroral electrojet index (AE index) during geomagnetically disturbed periods. The study also explores the suitability of the online sequential version of the best machine-learning algorithm, which has the potential for real-time forecast of the AE index from short-time input datasets with extremely fast convergence than batch-training methods. The study discusses the need for the correct choice of the input dataset that can be used for predicting the AE index from several combinations of input datasets which include coupling functions, geomagnetic indices and solar wind parameters. The study reveals that extreme learning machine and its online sequential version are promising models which could predict the AE index extremely fast with a high degree of accuracy even during disturbance periods. The study also shows that the choice of the polar cap index (PC index) as an input parameter is extremely important for an accurate prediction of the AE index.

Keywords. Machine learning; AE index.

\section{Introduction}

Forecasting the effects of solar-terrestrial phenomena and related magnetospheric activity on the near-Earth space is considered as one of the foremost topics of space research. Since the current technology related to solar-terrestrial investigations is vulnerable to magnetic storms or substorms, predicting geomagnetic activity well in advance is the primary aspect of space weather studies. Satellites, space stations, power grids, communication and navigation systems and airline controls are highly affected by solar disturbances and hence their predictions are of utmost importance. Time-series prediction is a major area in solar-terrestrial physics that has been intriguing the space weather modelling community over the past three decades (Baker et al. 1990; Hernandez et al. 1993; Klimas et al. 1997; Boberg et al. 2000; Gleisner and Lundstedt 2001; Amariutei and Ganushkina 2012). The main focus is to collect observations regarding major physical variables and analyse their relations to manifest a model that could describe future states of the system. Hence, according to time-series prediction, the forecasting algorithm needs an existing time-series data, say a sequence $\ldots X_{t-2}, X_{t-1}$ that predicts the future data values, say $X_{t}, X_{t+1}, \ldots$ such that the 
predicted values will have least statistical error when compared with the actual values reported at those time instants $t,(t+1)$, etc.

Iyemori et al. (1979) have introduced the linear prediction filtering technique in the magnetosphere according to which they proposed that a solar wind impulse and a geomagnetic disturbance response can be described by a linear time invariant filter. Soon it was extensively studied with a view to get an exact perspective on solar windmagnetosphere-ionosphere interactions by several researchers who later revealed that the so-called coupling processes contain several components of which one could be nonlinear (Clauer et al. 1981; Bargatze et al. 1985; McPherron et al. 1988). Later investigations have used nonlinear strategies for the prediction of geomagnetic activity such as establishing nonlinear dynamical models and using nonlinear prediction filtering algorithms (Baker et al. 1990; Klimas et al. 1992; Price and Prichard 1993; Vassiliadis et al. 1995). Klimas et al. (1997) have introduced a strategy for constructing analogues of physical systems that can accept input time series to produce an output series which is based on a local linear prediction model and can be manifested as an expression related to the autoregressive moving average (ARMA) filter. The conventional linear time-series models were created using statistical measures as building blocks and have certain advantages such as a simple feature selection and better expansion ability but have performance issues when nonlinearity and/or complexity appear in the system. These models have certain drawbacks such as their unsuitability for predicting output variables which are dependent on several physical parameters and there exist nonlinear relationships with the aforesaid parameters. For the past two decades, artificial neural networks (ANNs) have been employed as a promising alternative approach for time-series prediction and modelling (Lundstedt and Wintoft 1994; Kugblenu et al. 1999; Munsami 2000; Cai et al. 2010; Revallo et al. 2014; Unnikrishnan 2014). ANNs are advanced nonlinear dynamical systems having interconnected parallel computational elements arranged in predefined patterns that possess mesmerising adaptability to any kind of time series, low processing time, marvelous error tolerance and reasonable robustness. Its architecture is similar to biological neurons and is modelled after the human brain.

The fluctuation of the auroral current system gets constantly mapped into the variations of the auroral electrojet index called the AE index. The auroral current system is mainly composed of two parts such as the eastward electrojet (tracked by the AU index) and the westward electrojet (monitored by the AL index) currents that correspond to the convection electrojets (DP2 current system) and the substorm electrojets (DP1 current system), respectively (Akasofu 1975, 2004; Clauer and Kamide 1985; Rostoker et al. 1988). ANNs have been later introduced into the forecasting of AE indices along with nonlinear models such as the moving average and ARMA filters. Hernandez et al. (1993) used solar wind $v B_{\mathrm{S}}$ (where $v$ is the solar wind velocity and $B_{\mathrm{S}}$ is the interplanetary magnetic field (IMF) southward component) as one of the inputs to such an ANN model to predict the AL index. Gleisner and Lundstedt (1997) used ANN to predict the AE index from solar wind parameters such as solar wind density $(n)$, plasma speed $(V)$, IMF $B_{y}$ and IMF $B_{z}$. They used the temporal length of the input solar wind parameters that varies from 20 to 100 min which makes the number of input nodes in the input layer so large. Takalo and Timonen (1997) also tried to predict the AE index using the ANN with the solar wind electric field and previously observed AE index values. They reported that the prediction of the $\mathrm{AE}$ data is possible only for a period of $25 \mathrm{~min}$ ahead. Weigel et al. (1999) forecasted the auroral activity from solar wind inputs using neural networks. Gavrishchaka and Ganguli (2001) employed support vector machines (SVMs) to model the substorm activity reflected by the AE index. Gleisner and Lundstedt (2001) also reported the usage of dynamical neural networks for the prediction of the $\mathrm{AE}$ index but these networks also require a large number of input nodes. These earlier works clearly showed the potential of ANNs to predict the auroral activity.

Gleisner and Lundstedt (1999) also discussed the influence of ring currents on AE predictions. Even though they could not find much improvement in predicting the $\mathrm{AE}$ index with the Dst index by using an ANN gradient-descent back-propagation model, they suggested that Dst can partly compensate for the missing data on solar wind. They also indicated that using the Dst index is of potential importance in the operational forecasting of the $\mathrm{AE}$ where gaps in the stream of real-time solar-wind data are a common occurrence. Neural network techniques were also used to predict the Dst index from auroral indices (Stepanova and Pérez 2000 and references therein). Recently, Lazzús et al. 
(2016) have tried to predict the Dst index from the AE indices using time-delay neural network optimised with particle swarm algorithm.

For forecasting the AE index at different time scales, Pallocchia et al. (2008) used $V_{x}$ and IMF $B_{z}$ as inputs for the Elman ANN employed for their analysis. They have also reported that reasonable AE forecasts cannot be obtained by employing IMF only. The auroral geomagnetic activity comprises both solar wind 'directly' driven and loadingunloading processes (Rostoker et al. 1987 and references therein). In an extensive effort to forecast the hourly averaged AE index, Amata et al. (2009) have confirmed that the solar wind 'directly' driven $\mathrm{AE}$ component can be better forecasted than the unloading component, using solar wind parameters alone. Hence, it is certain that for reproducing the geomagnetic variations at auroral latitudes, geomagnetic indices must also be employed along with solar wind parameters. Recently, an ARMA model with exogenous inputs (ARMAX) model has been used by Amariutei and Ganushkina (2012) to predict the time variations of the AL index.

In the present work, a comparative analysis of different machine-learning algorithms such as feedforward back-propagation (FFBP) neural networks, SVMs, random forests (RFs) and extreme learning machines (ELMs) are used to predict the auroral index (AE index) from certain geomagnetic indices, solar wind parameters and coupling functions. The prediction analysis also focuses on the choice of a suitable coupling function that could replace the $\mathrm{PC}$ index in predicting the $\mathrm{AE}$ index. The Dst index, solar wind velocity component $\left(V_{x}\right)$ and the north-south component of the IMF $\left(B_{z}\right)$ are chosen as constant input parameters in all models.

\section{Data}

The 1-min AE index has been taken from the World Data Center for Geomagnetism, Kyoto, Japan. The USGS Dst index (which is a 1-min alternative of the hourly Kyoto-Dst index, available through the USGS database) used for the present analysis is based on the geomagnetic $\mathrm{H}$-component from a few low-latitude magnetic observatories such as Honolulu, Kakioka, San Juan and Hermanus (Gannon and Love 2011). Other geomagnetic data such as SYM-H, SYM-D, ASY-H and ASY-D indices are also available through World Data Center for Geomagnetism, Kyoto, Japan while the PC index is available through the World Data Center for Geomagnetism, Copenhagen. Solar wind and IMF data from the 1-min high-resolution NASA OMNI database have been taken, which transform solar wind parameters to GSM coordinates and time shifted (using the solar wind velocity measurement) to the location of the Earth's upstream magnetopause (King and Papitashvili 2005).

\section{Methods}

The methods that are used in the present work for the comparative analysis of machine-learning methods for the prediction of the $\mathrm{AE}$ index are ELMs, SVMs, random forests and feedforward back-propagation ANNs.

\subsection{Extreme learning machines}

ELM and its variants are novel machine-learning algorithms proposed for training single hidden layer feedforward neural networks (FFNNs) to solve regression problems (Huang et al. 2006). The major advantage of ELMs is that they randomly choose input weights, say $W$, and biases, say $\alpha$. For a set of fixed input weights, an independent set of output weights, say $\beta$, is computed such that the training focuses on achieving a solution to $\beta$. Such a solution will also be linear for a linear output layer thus featuring an extremely fast learning procedure than for most popular ANN algorithms such as feedforward back-propagation. ELMs have also demonstrated excellent generalisation ability than conventional ANNs (Huang et al. 2011).

Given $N$ distinct samples comprising a dataset $D=\left\{\left(x_{i}, y_{i}\right)\right\}_{i=1}^{N}$, where $x_{i} \in \mathbb{R}^{n}$ with $x_{i}=$ $\left[x_{i 1}, x_{i 2}, \ldots, x_{i n}\right]^{\mathrm{T}}$ and $y_{i} \in \mathbb{R}^{m}$ with $y_{i}=\left[y_{i 1}, y_{i 2}\right.$, $\left.\ldots, y_{i m}\right]^{\mathrm{T}}$, then ELM with $L$ hidden additive nodes and activation function $g(\cdot)$ can be expressed as:

$$
\begin{aligned}
f\left(x_{j} ; w, \alpha, \beta\right) & \equiv \sum_{i=1}^{L} \beta_{i} g\left(w_{i} \cdot x_{j}+\alpha_{i}\right) \\
& =y_{j}, j=1, \ldots, N
\end{aligned}
$$

where $w_{i}=\left[w_{i 1}, w_{i 2}, \ldots, w_{i n}\right]^{\mathrm{T}}$ is the vector of weights that connects the $i$ th hidden node while the input nodes, $\beta_{i}=\left[\beta_{i 1}, \beta_{i 2}, \ldots, \beta_{i m}\right]^{\mathrm{T}}$ is the vector of weights that connects the $i$ th hidden node and the output nodes and $\alpha_{i}$ signifies the threshold of the $i$ th hidden node. If $h(x)$ is a function that maps the data from the $d$-dimensional input 
space to the $L$-dimensional ELM feature space, then $h(x)=\left[g\left(w_{1}, \alpha_{1}, x\right), \ldots, g\left(w_{L}, \alpha_{L}, x\right)\right]$ where $g(w, \alpha, x)$ can also be a nonlinear piecewise continuous function and $\left\{\left(w_{i}, \alpha_{i}\right)\right\}_{i=1}^{L}$ is a set of randomly generated values that belong to a continuous probability distribution function (Huang et al. 2015). For the sigmoid function, it can be described as $(w, \alpha, x)=1 /(1+\exp [-(w \cdot x+\alpha)])$. Equation (1) can also be represented in the simplest form as:

$$
H \beta=y,
$$

where the hidden layer $H$ is denoted by

$$
H=\left[\begin{array}{ccc}
g\left(w_{1} \cdot x_{1}+\alpha_{1}\right) & \cdots & g\left(w_{L} \cdot x_{1}+\alpha_{L}\right) \\
\vdots & \ddots & \vdots \\
g\left(w_{1} \cdot x_{N}+\alpha_{1}\right) & \cdots & g\left(w_{L} \cdot x_{N}+\alpha_{L}\right)
\end{array}\right]
$$

and $\beta=\left[\beta_{1}, \beta_{2}, \ldots, \beta_{L}\right]^{\mathrm{T}}$ denotes the matrix of output weights and $y=\left[y_{1}, y_{2}, \ldots, y_{m}\right]^{\mathrm{T}}$ denotes the matrix of targets. Hence, to summarise, it can be said that the goal of the ELM is to minimise the training error $\left(\|H \beta-y\|^{2}\right)$ as well as the norm of the output weights $(\|\beta\|)$.

When the conventional ELM is used, all the training data has to be fed prior to training. But in real applications, such as solar-terrestrial analysis, the training data may arrive only one after the other or as chunks. Liang et al. (2006) presented a sequential learning algorithm called online sequential extreme learning machine (OS-ELM), which can handle these chunks of data and use them for training the network. In OS-ELM having additive nodes, the input weights as well as biases were randomly created, and based on this, the output weights are determined in an analytical fashion. Unlike other sequential learning algorithms, OSELM also requires only the number of hidden nodes to be specified as the conventional ELM (Jia et al. 2016).

The major features of OS-ELM can be elucidated as:

(1) The training data are sequentially (one after the other with varying or fixed block length) presented to the learning algorithm.

(2) At any instant, only the newly arrived fresh block of observations are fed and learned instead of supplying the entire past data.

(3) A block of training data is discarded as soon as the learning sequence for that block of observation has been completed.
(4) The training algorithm will not have any prior knowledge about the number of training observations that will be presented.

ELM does not require any parameter tuning except that the number of hidden neurons as well as the kernel function should be properly selected. This makes ELM one of the easiest networks to implement among the machine-learning networks. For OS-ELM, the number of initial training data used in the initial phase of OS-LEM is 1000 with the size of block of data learned by OS-ELM in each step being 50. For further details regarding OSELM, the readers may refer to the work of Liang et al. (2006).

\subsection{Support vector machines}

SVMs are regressor machine-learning algorithms, also called support vector regression (SVR) algorithms that perform data correlation through nonlinear mapping (Drucker et al. 1997; Vapnik 1998). The primary advantage of SVRs is that both linear and nonlinear kernel functions can be used to solve regression problems. The versatility of the SVRs is associated with the collection of kernel functions that can be used which convert the input space to a higher-dimensional output feature space. Such a mapping will ensure that the results in the output space will certainly correspond to the results of the actual low-dimensional input space. Thus, SVR employs an easy way of calculating the inner product in a feature space which is directly available as a function of the original input points (Vapnik and Chapelle 2000).

Suppose a training data of the form $\left\{\left(x_{i}, y_{i}\right)\right\}_{i=1}^{N}$ $\subset S \times \mathbb{R}$ has been used where $S \rightarrow \mathbb{R}^{\mathrm{d}}$ denotes the input space. The primary goal of SVR is to find a maximal flat function $f(x)$ which has $\varepsilon$-deviation at most from the actually obtained targets $y_{i}$ for all the training data. Hence, those errors which are less than $\varepsilon$ will be accepted but those that are larger than the threshold will be rejected. If the case of linear functions of the form $f(x)=\langle w, x\rangle+b$ are considered with $w \in S, b \in \mathbb{R}$, where $\langle.,$.$\rangle denotes$ the dot product in $S$ such that a small $w$ is preferred by minimising the norm $\langle w, w\rangle=\|w\|^{2}$. The goal of the SVR is to minimise $(1 / 2)\|w\|^{2}$ subject to $y_{i}-\left\langle w, x_{i}\right\rangle-b \leq \varepsilon$ and $\left\langle w, x_{i}\right\rangle+b-y_{i} \leq \varepsilon$. Such an assumption is possible only if such a function $f$ actually exists that approximates all pairs $\left(x_{i}, y_{i}\right)$ with a precision of $\varepsilon$. But, if the problem demands an increase in error margin, then slack variables 
$\left\{\xi, \xi^{\prime}\right\}$ have been introduced to modify the infeasible constraints of the optimisation problem. The modified optimisation problem can be written as:

$$
\begin{aligned}
& \text { minimise } C \sum_{i=1}^{N}\left(\xi_{\mathrm{i}}+\xi_{i}^{\prime}\right)+\frac{1}{2}\|w\|^{2} \\
& \text { subject to }\left\{\begin{array}{l}
y_{i}-\left\langle w, x_{i}\right\rangle-b \leq \varepsilon+\xi_{i}, \\
\left\langle w, x_{i}\right\rangle+b-y_{i} \leq \varepsilon+\xi_{i}^{\prime}, \\
\xi, \xi^{\prime} \geq 0,
\end{array}\right.
\end{aligned}
$$

where the cost parameter $C>0$ signifies the compromise between the empirical risk minimisation and flatness of the model function.

For using SVR, several parameters need to be tuned. One of them is the cost parameter $C$ which is taken as 1 . Higher values of $C$ tend to give a predicted series having higher amplitudes than the actual series. The $\varepsilon$ in the loss function is set at the value 0.01 which gives the best performance. In the present analysis, SVR using the kernel function (nonlinear SVR) called radial basis function (RBF) has been used. For RBF kernel, gamma is taken as $1 / \zeta$ where $\zeta$ is the number of features. For all other parameters, default values provided in the LIBSVM package have been used.

\subsection{Random forests}

Decision trees can be considered as one of the popular and simple methods for performing machine-learning tasks, of which its reformed variant called the random forest model, can be regarded as an efficient ensemble learning algorithm that can handle high-dimensional regression problems (Breiman 2001). Breiman (1996) proposed the bootstrap aggregating method (also called as bagging) to reduce prediction variance for classification and regression trees algorithm which had been introduced earlier by Breiman et al. (1984). A RF is an ensemble of different regression trees where each tree plays the role of a nonlinear mapping function that maps complex input spaces into continuous output spaces. The basic functioning of RF is based on a set of rules that perform certain tests based on the fact that a parent node in the RF tree maintains a decision that is enforced on a data sample to branch it through either the left or the right daughter node. Even though a standard single decision tree is prone to over-fitting, an ensemble of randomly trained trees having high generalisation ability usually produces better results (Dietterich 2000; Geurts et al. 2006).

The basic RF algorithm is built on three fundamental steps:

(1) In the first step, the algorithm draws $N$ bootstrap samples from the actual data.

(2) For each bootstrap sample, grow a regression tree such that at each node, rather than taking the best split among all predictors, the algorithm randomly samples $m(0<m \leq q)$ of the $q$ predictors and from those variables, it chooses the best split.

(3) In the final step, the algorithm predicts new data by averaging the predictions of the $N$ trees.

For forecasting an observation with predictor values, say $x$, let $\hat{h}_{n}(x)$ denote the prediction from the $n$th tree in the forest, $n=1, \ldots, N$. The $\mathrm{RF}$ method uses the average of the individual predictions from trees to establish the final result:

$$
\hat{h}_{\mathrm{RF}}=\frac{1}{N} \sum_{n=1}^{N} \hat{h}_{n}(x)=\operatorname{mean}\left\{\hat{h}_{n}(x)\right\} .
$$

For more details regarding the RF algorithm and equations, the reader is referred to the book of Pavlov (1997). For tuning random forests, the parameter that seems to be sensitive is $m$, which is the number of randomly assigned predictor variables at each node. For regression applications, usually $m$ is taken as one-third of the total number of predictor variables. For the present study, $m$ is taken as the number of inputs (or features) divided by three in each case. The number of trees grown in the forest is taken as 1000 . The maximum number of terminal nodes (tree size) is taken as 5 .

\subsection{FFBP neural networks}

The FFNN is the simplest type of ANN in which the information moves only in the forward direction, from the input nodes through the hidden nodes into the output nodes (Anderson et al. 1988). Later, it was proposed that, to improve the efficiency of the network, the errors of the network have to be propagated backwards which results in an enhanced ANN architecture called as the FFBP neural network. The training of the FFBP neural network is mainly carried out using the back-propagation (BP)-based learning algorithms (Rumelhart and McClelland 1986; Hammerstrom 1993). The architecture of this network consists of 
an input layer, hidden layers and output layers. When modelling time-series data with nonlinear data, usually the three-layered FFBP network is the widely used model. A number of different kinds of BP learning algorithms (other than the conventional BP algorithm) have been proposed, such as resilient back-propagation (RBP), RBP with or without weight backtracking, RBP with globally convergent training scheme, etc. (Riedmiller and Braun 1993; Riedmiller 1994; Anastasiadis et al. 2005).

To improve certain drawbacks of $\mathrm{BP}$ algorithm such as its slow convergence, second-order algorithms like the Gauss-Newton method and the Levenberg-Marquardt (LM) algorithm have been preferred over first-order algorithms (Liu 2010 and references therein). Second-order algorithms use a relatively fast Hessian matrix approximation scheme using less complex Jacobian matrix computations to produce better estimations on both directions as well as step sizes which significantly improves the convergence time. The LM algorithm combines the low training time of the GaussNewton algorithm as well as the stability of the conventional BP algorithm called gradient-descent, which makes LM the most efficient algorithms for training moderate-size patterns. The feedforward layer network is created such that it takes a vector of $N$ hidden layer sizes and a BP training function while returns an FFNN with $N+1$ layers. For network training, if LM is used to update weight and bias values, then it is seen that the network time is significantly improved (Liu 2010). The changes during the training period are under the influence of the transfer function used. When the performance goal has been met after a particular number of epochs, it marks the completion of the training phase. The maximum number of epochs is taken as 1000 with a performance goal of 0.01 .

For predicting the AE index, six sets of training data have been used with each having a time span of 1 month, January 2017. The period is chosen because the solar minimum year has few data gaps in solar wind data compared with solar maximum years. The period of forecast is the first day of February 2017. The forecast day also marks a minor geomagnetic storm beginning on 31 January through 1 February 2017 (UTC days) due to

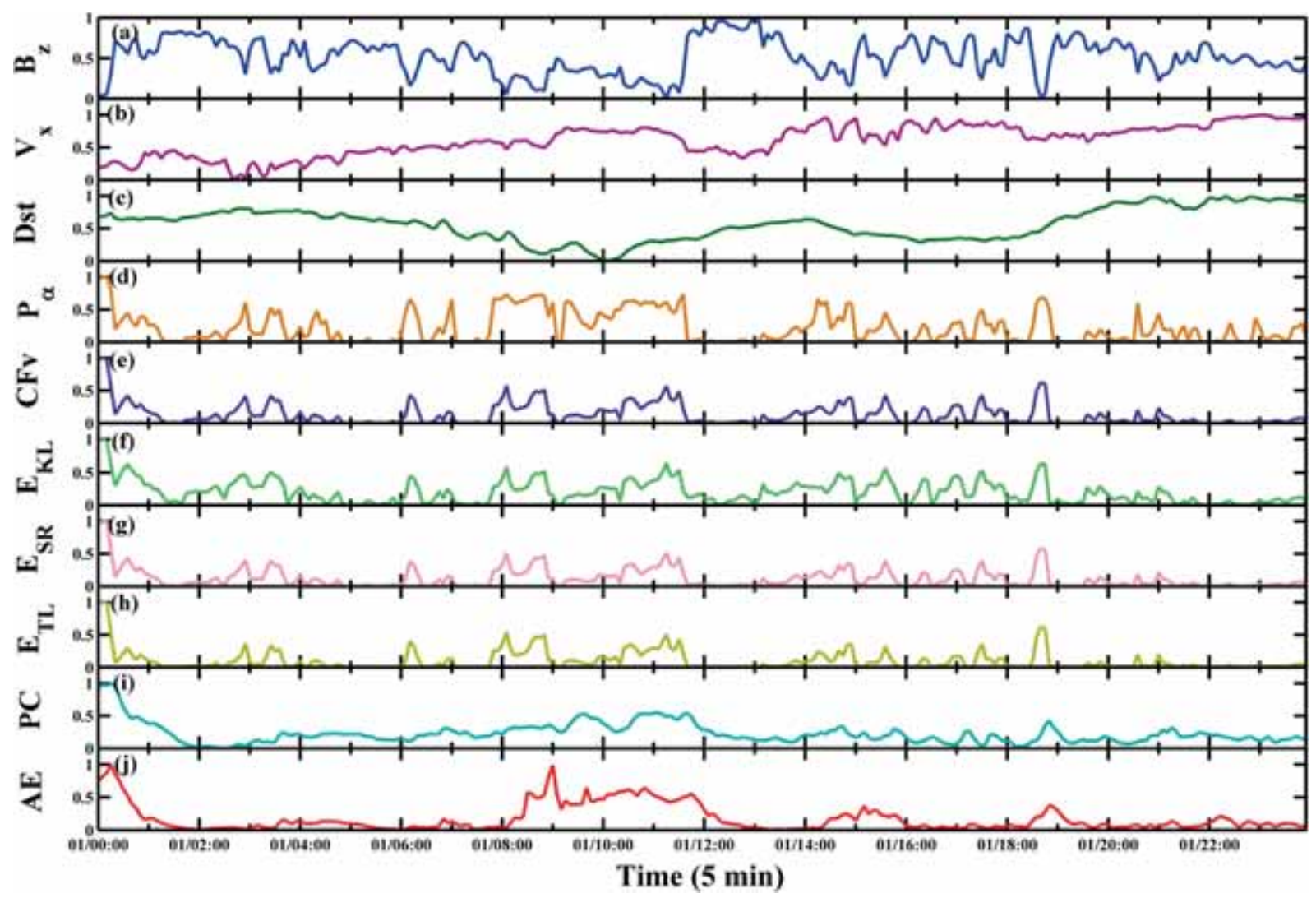

Figure 1. Temporal variations of input and output parameters used in SETS 1-6 during the testing day 1 February 2017 where (a) $\operatorname{IMF} B_{z},(\mathbf{b}) V_{x}$, (c) Dst, (d) $P_{\alpha},(\mathbf{e}) \mathrm{CF}_{v},(\mathbf{f}) E_{\mathrm{KL}},(\mathbf{g}) E_{\mathrm{SR}},(\mathbf{h}) E_{\mathrm{TL}},(\mathbf{i}) \mathrm{PC}$ are the input data and $(\mathbf{j})$ AE are the output data. All the data are in normalised units and temporal resolution of all data are 5 min. 
recurrent, negative polarity high-speed stream from coronal holes. Thus, each model will take 1 month data (January 2017) for training to predict the AE index of a disturbance day, namely, 1 February 2017. All datasets are normalised and the data gaps are filled with the linear interpolation technique. The coupling functions used are Kan-Lee electric field $\left(E_{\mathrm{KL}}\right)$, Scurry-Russel function $\left(E_{\mathrm{SR}}\right)$, Vasyliunas coupling function $\left(\mathrm{CF}_{v}\right)$, Temerin and $\mathrm{Li}$ function $\left(E_{\mathrm{TL}}\right)$ and Finch and Lockwood function $\left(P_{\alpha}\right)$. From earlier studies, it is seen that these coupling functions have a higher nonlinear correlation with the PC index (Gopinath et al. 2015). For detailed expressions or equations regarding the
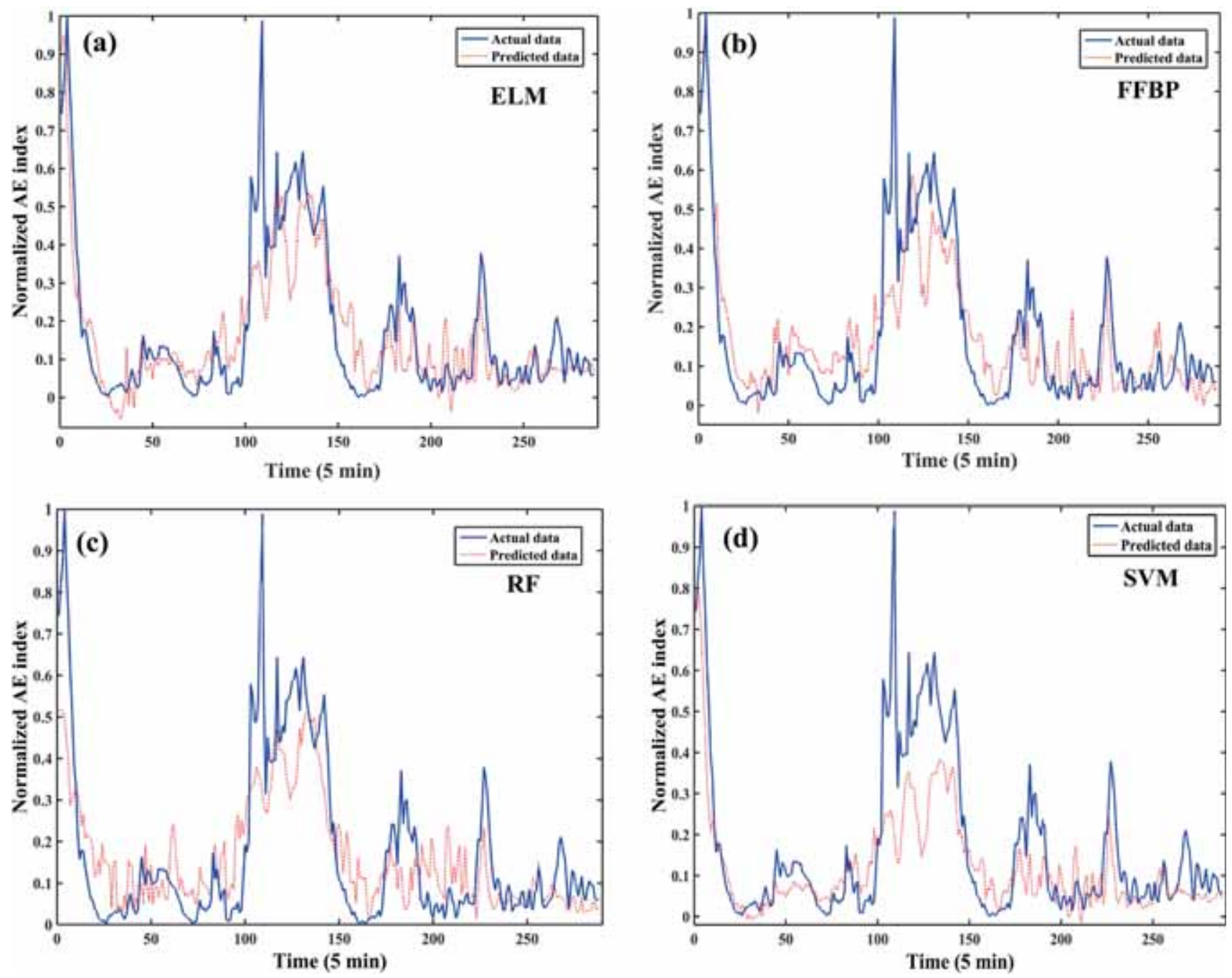

Figure 2. Actual (blue bolded) and predicted data (red dotted) from different models using SET 1 for the geomagnetic storm day on 1 February 2017: (a) ELM model, (b) FFBP model, (c) random forest (RF) model, and (d) SVM model.

Table 1. Statistical analysis of SET 1 used for the prediction of the AE index on 1 February 2017.

\begin{tabular}{lrcccc}
\hline Model & $\begin{array}{c}\text { Training } \\
\text { time (s) }\end{array}$ & $\begin{array}{c}\text { Testing } \\
\text { time (s) }\end{array}$ & RMSE & ARV & $R$ \\
\hline ELM (sigmoid) & 0.0624 & 0.012 & 0.1079 & 0.2854 & 0.8351 \\
OS-ELM (sigmoid) & 0.2340 & 0.0156 & 0.1080 & 0.2861 & 0.8347 \\
Random forest & 164.7839 & 0.0780 & 0.1246 & 0.3808 & 0.7800 \\
FFBP (LM) & 8.2213 & 0.0312 & 0.1105 & 0.2992 & 0.8271 \\
SVM (RBF) & 8.4865 & 0.1248 & 0.1307 & 0.4184 & 0.7583 \\
\hline
\end{tabular}


coupling functions, the readers are referred to the paper of Gopinath et al. (2015) and the references therein.

Training input datasets:

(1) SET 1: Dst and PC indices $+\mathrm{SW}$ parameters (velocity $V_{x}$ and IMF $B_{z}$ ).

(2) SET 2: Dst index + coupling function $P_{\alpha}+$ SW parameters (velocity $V_{x}$ and IMF $B_{z}$ ).

(3) SET 3: Dst index + coupling function $\mathrm{CF}_{v}+$ SW parameters (velocity $V_{x}$ and $\operatorname{IMF} B_{z}$ ).

(4) SET 4: Dst index + coupling function $E_{\mathrm{KL}}+$ SW parameters (velocity $V_{x}$ and IMF $B_{z}$ ).

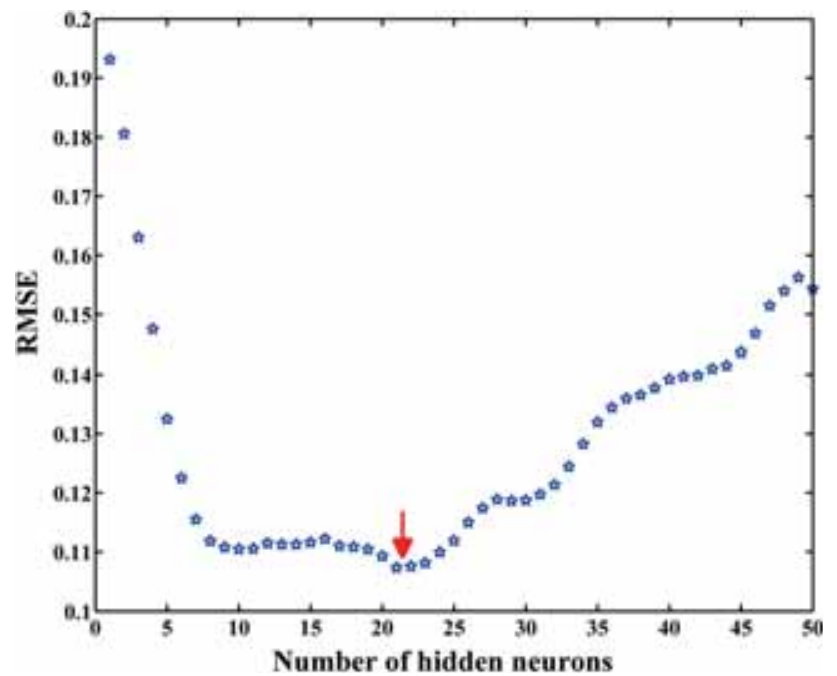

Figure 3. Optimisation of the number of hidden neurons for the ELM model (SET 1). The number of hidden neurons (22) where RMSE drops to minimum (marked with a red arrow) is considered for the analysis.

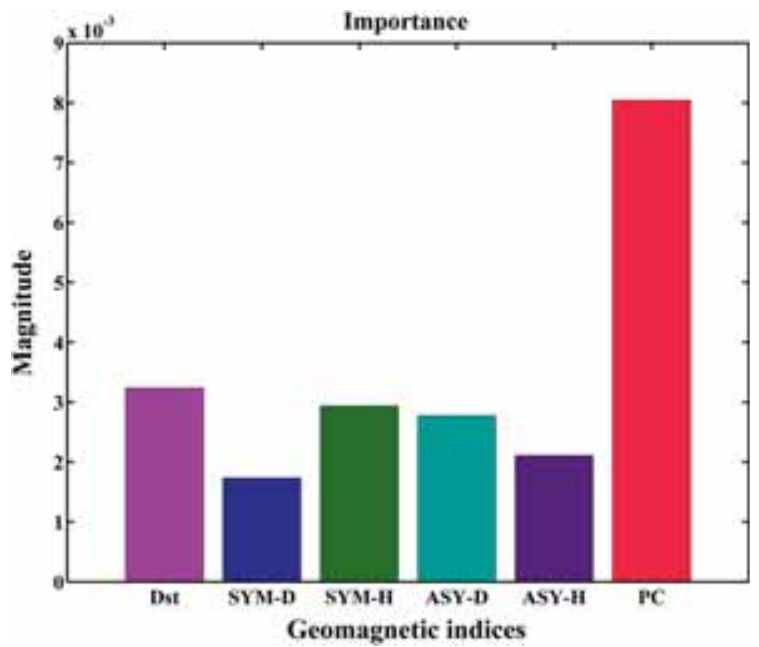

Figure 4. Importance of various geomagnetic indices such as Dst, SYM-D, SYM-H, ASY-D, ASY-H and PC in predicting the AE index as given by the random forest model.
(5) SET 5: Dst index + coupling function $E_{\mathrm{SR}}+$ SW parameters (velocity $V_{x}$ and IMF $B_{z}$ ).

(6) SET 6: Dst index + coupling function $E_{\mathrm{TL}}+$ SW parameters (velocity $V_{x}$ and IMF $B_{z}$ ).

The forecasting performances of all the models are evaluated in terms of well-known error statistics such as the root mean squared error (RMSE), average relative variance (ARV) and $R$ value. If $y_{i}$ denotes predicted series and $Y_{i}$ the actual series, they can be defined as

$$
\begin{gathered}
\mathrm{RMSE}=\sqrt{\frac{\sum_{i=1}^{n}\left(y_{i}-Y_{i}\right)^{2}}{n},} \\
\mathrm{ARV}=\frac{\sum_{i=1}^{n}\left(y_{i}-Y_{i}\right)^{2}}{\sum_{i=1}^{n}\left(\operatorname{mean}\left(Y_{i}\right)-Y_{i}\right)^{2}}, \\
R=1-\left|\frac{\sum_{i=1}^{n}\left(y_{i}-Y_{i}\right)^{2}}{\sum_{i=1}^{n} Y_{i}}\right| .
\end{gathered}
$$

\section{Results and discussion}

The different training sets as well as the corresponding testing sets are fed into each model to compare their performance. The temporal variations of the input and the output parameters for SETS 1-6 during 1 February 2017 are shown in figure 1. The comparison between the predicted and actual AE indices from SET 1 inputs for each

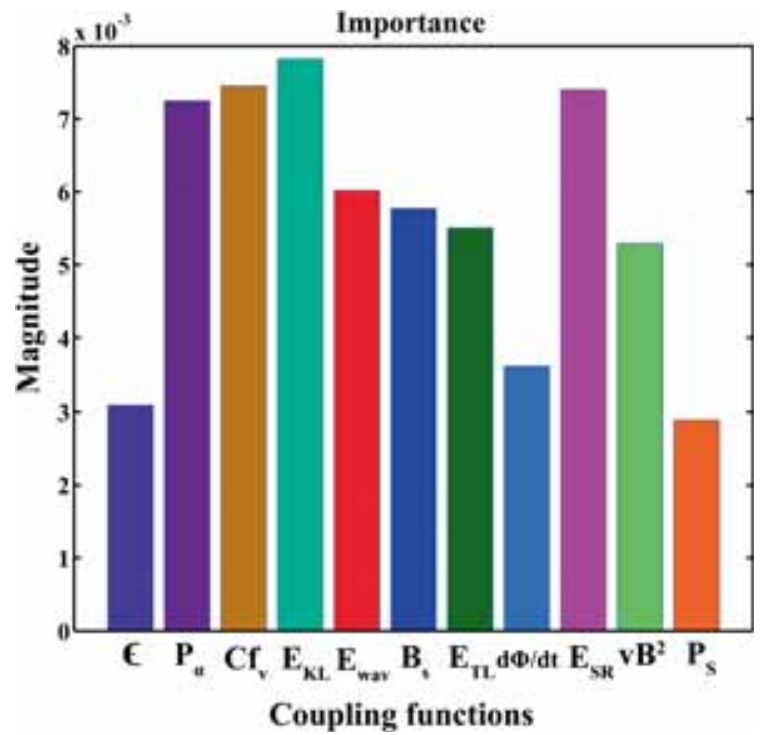

Figure 5. Importance of various coupling functions in predicting the $\mathrm{AE}$ index as given by the random forest model. 
Table 2. Statistical analysis of SETS 2-6 used for the prediction of the AE index on 1 February $201 \%$.

\begin{tabular}{llrllll}
\hline \multirow{2}{*}{ Dataset } & \multicolumn{1}{c}{ Model } & $\begin{array}{c}\text { Training } \\
\text { time (s) }\end{array}$ & $\begin{array}{l}\text { Testing } \\
\text { time (s) }\end{array}$ & RMSE & ARV & $R$ \\
\hline SET 2 & ELM (sigmoid) & 0.0468 & 0.0312 & 0.1636 & 0.6559 & 0.6211 \\
& Random forest & 171.4295 & 0.0780 & 0.1879 & 0.8651 & 0.5003 \\
& FFBP (LM) & 11.9029 & 0.0314 & 0.1788 & 0.7840 & 0.5471 \\
\multirow{2}{*}{ SET 3 } & SVM (RBF) & 7.9873 & 0.1248 & 0.1632 & 0.6532 & 0.6227 \\
& ELM (sigmoid) & 0.0624 & 0.0312 & 0.1738 & 0.7408 & 0.5721 \\
& Random forest & 172.9895 & 0.0780 & 0.1853 & 0.8417 & 0.5138 \\
& FFBP (LM) & 13.8529 & 0.0313 & 0.1705 & 0.7128 & 0.5882 \\
SET 4 & SVM (RBF) & 7.9873 & 0.1092 & 0.1820 & 0.8115 & 0.5312 \\
& ELM (sigmoid) & 0.0624 & 0.0312 & 0.1526 & 0.5707 & 0.6703 \\
& Random forest & 179.6508 & 0.0936 & 0.1819 & 0.8113 & 0.5313 \\
& FFBP (LM) & 7.1916 & 0.0312 & 0.1665 & 0.6794 & 0.6075 \\
SET 5 & SVM (RBF) & 8.6113 & 0.1404 & 0.1781 & 0.7772 & 0.5510 \\
& ELM (sigmoid) & 0.0624 & 0.0312 & 0.1738 & 0.7401 & 0.5724 \\
& Random forest & 166.9991 & 0.0780 & 0.1862 & 0.8501 & 0.5089 \\
& FFBP (LM) & 11.0293 & 0.0314 & 0.1767 & 0.7654 & 0.5578 \\
SET 6 & SVM (RBF) & 8.2525 & 0.1248 & 0.1782 & 0.7781 & 0.5505 \\
& ELM (sigmoid) & 0.0468 & 0.0312 & 0.1617 & 0.6411 & 0.6296 \\
& Random forest & 165.0335 & 0.0780 & 0.1833 & 0.8234 & 0.5244 \\
& FFBP (LM) & 13.0729 & 0.0312 & 0.1658 & 0.6740 & 0.6106 \\
& SVM (RBF) & 8.0341 & 0.1092 & 0.1780 & 0.7765 & 0.5515 \\
\hline
\end{tabular}

model is shown in figure 2. The details of the statistical comparison of the models for SET 1 are shown in table 1 . After analysing the results, it is certain that the ELM model shows the lowest RMS test error, the lowest ARV and the highest $R$ value than other models for SET 1. It is also amazing to see that ELM model produces the result in the lowest network time (sum of training and testing time). The statistical values of the OS-ELM model are also comparable to the values of the conventional ELM model. Hence, an inference can be drawn that for the SET 1, the ELM model is superior to other models as it gives a better prediction of the AE index. The optimisation of the number of hidden neurons for the ELM model (for SET 1) is shown in figure 3. Although not shown, a similar optimisation strategy is adopted for the FFBP model also for optimising the hidden layer size $N$. The importance of different predictors (of different geomagnetic indices such as Dst, SYM-H, ASY-H, SYM-D, ASY-D and PC) in predicting the $\mathrm{AE}$ index, with the importance graph derived from the random forest is shown in figure 4 . The graph shows that both the Dst and PC indices have more importance than other geomagnetic indices. The graph also shows the importance of the PC index which is by far high than any other geomagnetic index and can be considered as quite contributing to the fluctuations of the AE index. This is not a surprising result as the $\mathrm{PC}$ index can also be considered as a quantifier that tracks the energy that penetrates into the polar magnetosphere during solar wind-magnetosphere coupling (Takalo and Timonen 1999). Even though the important estimation generated by the random forest model may not reflect the actual significance of predictors considered by other models, it can be considered as a basic selection criterion for the choice of input parameters.

The SETS 2-6 replace the PC index with coupling functions $P_{\alpha}, \mathrm{CF}_{v}, E_{\mathrm{KL}}, E_{\mathrm{SR}}$ and $E_{\mathrm{TL}}$, respectively. Figure 5 shows the importance of coupling functions as predictors in forecasting the $\mathrm{AE}$ index. The details of the statistical comparison of the models for SETS 2-6 are shown in table 2. In this analysis, OS-ELM is not considered because while comparing the statistical values, there is not much of a difference in the prediction performance of both conventional ELM and OS-ELM. Table 2 shows that the best statistical values are for the SET 4 (coupling function $E_{\mathrm{KL}}$ ) using the ELM model. Figure 6 shows the comparison between the models in predicting the AE index using SET 4. The ELM shows extremely fast convergence with 

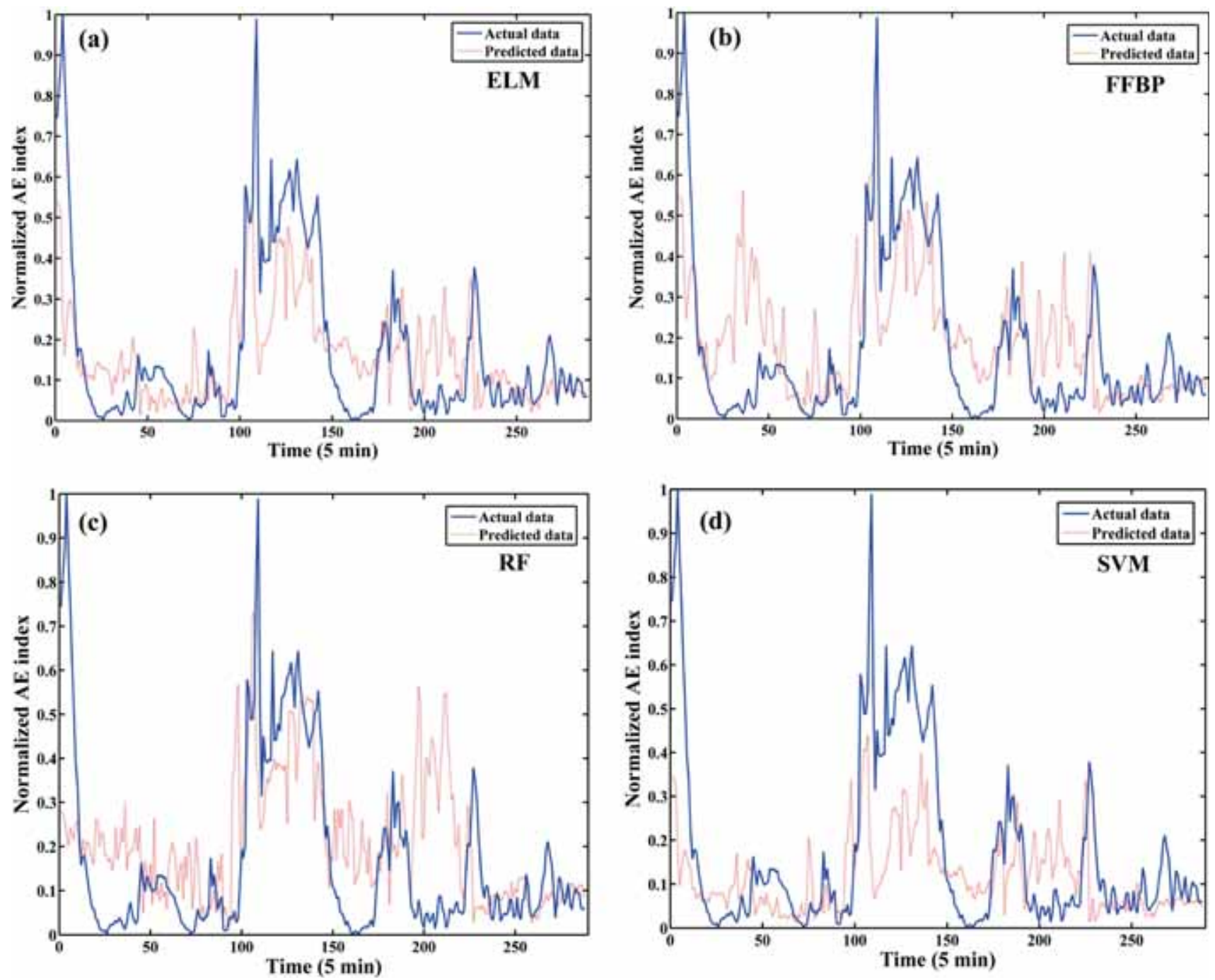

Figure 6. Actual (blue bolded) and predicted data (red dotted) from different models using SET 4 (set with a coupling function having the best statistical values) for the geomagnetic storm day on 1 February 2017: (a) ELM model, (b) FFBP model, (c) random forest (RF) model and (d) SVM model.

good error statistics for almost all sets. The FFBP model also shows appreciable statistics but it is slow compared with the ELM models. The SVM models show comparable statistics with the FFBP models in certain cases such as SET 2 or SET 5 but are slower than the FFBP models. Random forest models show extremely slow convergence than other models and also show poor statistics. The polar cap magnetic activity is closely associated with the PC index which is calibrated by the Kan-Lee field $\left(E_{\mathrm{KL}}\right)$ and is parameterised by season, universal time and hemisphere (Troshichev and Janzhura 2012). The analysis also reveals the importance of the $\mathrm{PC}$ index in predicting the $\mathrm{AE}$ index, since replacing the PC index with coupling functions showed that the latter could act as potential predictors only to a certain extent.
The prediction performance can be improved further only by introducing more geomagnetic indices as inputs or by developing a combination of other geomagnetic indices that correspond to those latitudes near the auroral zone and coupling functions that may have a strong relationship with transient fluctuations within the magnetosphereionosphere system in the high-latitude region. So the Dst index (which is an equatorial geomagnetic disturbance index) has been replaced with a set of mid-latitude geomagnetic indices called the SYM and ASY indices (such as SYM-D, SYM-H, ASY-D and ASY-H). The other inputs are IMF $B_{z}$ and $V_{x}$ and any one of the coupling functions $P_{\alpha}$, $\mathrm{CF}_{v}, E_{\mathrm{KL}}, E_{\mathrm{SR}}$ or $E_{\mathrm{TL}}$ fed at a time. Figure 7 shows the comparison between the predictions using the Dst index (figure 7a) and the mid-latitude 

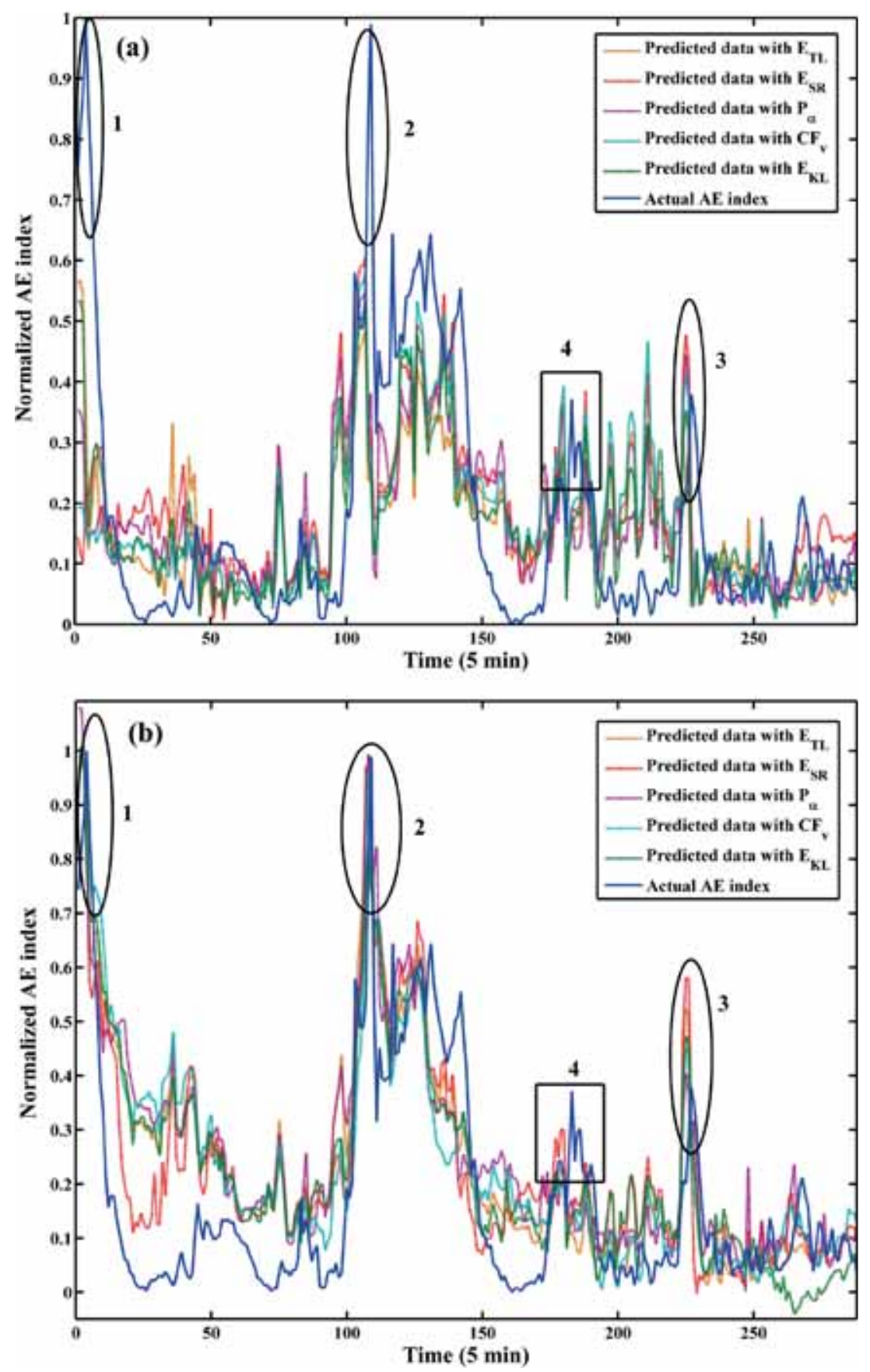

Figure 7. Predicted and actual AE indices for 1 February 2017 using the ELM model where (a) the prediction using the Dst index as one of the inputs, (b) replacing the Dst index with mid-latitude geomagnetic indices such as SYM-D, SYM-H, ASY-D and ASY-H indices. The other inputs are IMF $B_{z}, V_{x}$ and any one of the coupling functions $P_{\alpha}, \mathrm{CF}_{v}, E_{\mathrm{KL}}, E_{\mathrm{SR}}$ or $E_{\mathrm{TL}}$. The ellipses show the bursty regions in the AE index which are better predicted by the mid-latitude geomagnetic indices when compared with the Dst index while the square shows the bursty region which cannot be predicted well by both the Dst and mid-latitude indices.

geomagnetic indices (figure $7 \mathrm{~b}$ ) using the ELM model. In the figures, certain bursty regions of the AE index are marked as ellipses where the predictions using the Dst index have shown large deviations. It is clear from figure 7 (b) that those regions marked as ellipses are well predicted using mid-latitude indices. Also, the region marked by the square cannot be predicted successfully even by both the Dst and mid-latitude indices. The statistical values of the analysis using mid-latitude indices employing the ELM model are shown in table 3 . 
Table 3. Statistical analysis of using mid-latitude geomagnetic indices (replacing Dst) for the prediction of the AE index on 1 February 2017 with the ELM model.

\begin{tabular}{lccccc}
\hline Parameter & $\begin{array}{c}\text { Training } \\
\text { time }(\mathrm{s})\end{array}$ & $\begin{array}{c}\text { Testing } \\
\text { time }(\mathrm{s})\end{array}$ & RMSE & ARV & $R$ \\
\hline$P_{\alpha}$ & 0.0642 & 0.0312 & 0.1681 & 0.6925 & 0.6000 \\
$\mathrm{CF}_{v}$ & 0.0543 & 0.0322 & 0.1637 & 0.6573 & 0.6203 \\
$E_{\mathrm{KL}}$ & 0.0468 & 0.0341 & 0.1528 & 0.5721 & 0.6695 \\
$E_{\mathrm{SR}}$ & 0.0469 & 0.0312 & 0.1426 & 0.4988 & 0.7119 \\
$E_{\mathrm{TL}}$ & 0.0471 & 0.0325 & 0.1554 & 0.5917 & 0.6582 \\
$\mathrm{PC}$ index & 0.0342 & 0.0246 & 0.1081 & 0.2865 & 0.8345 \\
\hline
\end{tabular}

Other parameters are IMF $B_{z}$, solar wind velocity $V_{x}$, SYM and ASY indices.

The variable forcing of the solar wind streams and the propagation of IEF generate several fields and currents such as the magnetopause current, field-aligned (Birkeland) currents, cross-tail current, partial ring current (PRC) and the ring current in the magnetosphere-ionosphere system (Alexeev et al. 1996; Friedrich et al. 1999; Liemohn et al. 2001 and references therein). The relationships between the PRC and mid-latitude asymmetry indices were studied by Weygand and McPherron (2006). During magnetic storms, the increase in the dynamic pressure of the solar wind causes large variations in magnetopause current as well as magnetotail current. This in turn generates plasma instabilities in the magnetotail causing a substorm current wedge associated with the initiation of the unloading processes into the ionosphere. During substorms, auroral arcs suddenly brighten and heavy magnetic disturbance occurs at the auroral latitudes. In the mean time, geomagnetic disturbances also occur at the equatorial and mid-latitude regions due to the energisation and dissipation of the ring current. These processes jointly create the intermittency in the ionospheric sections of the aforesaid current systems which flow at auroral latitudes called AEs. Since the AE index reflects the fluctuations in the AEs, the prediction of the AE index becomes a challenging task during disturbance times. On these grounds, the present analysis also shows that the AE index is one of the toughest indices to predict as the signatures of internal dynamics generated by the loading-unloading processes and the solar wind driving conditions together create 'bursts' which are difficult to forecast with extreme accuracy even using solar wind and geomagnetic predictors.

\section{Conclusions}

A comparative analysis of several machine-learning paradigms has been shown for the prediction of the AE index. For that purpose, geomagnetic indices such as the Dst index, PC index, solar wind velocity $V_{x}$ and IMF $B_{z}$ and a few coupling functions have been employed. From these results, it is seen that the work clearly revealed the potential of the $\mathrm{PC}$ index in predicting the $\mathrm{AE}$ index. When compared with the Dst index, the predictive performance reveals the ability of the mid-latitude indices (like SYM and ASY indices) in predicting the fluctuations of the AE index in a better way. The analysis also shows that the ELM models show extremely fast convergence with the best statistical results. Another variant of the ELM model called the OS-ELM model is seen to be extremely fast in predicting the auroral index data in real time than batch-training machine-learning algorithms. The training data can be fed as chunks or one after the other as soon as they arrive and the trained data blocks can be erased soon after training is accomplished, which help the model run on networks even with low storage capabilities. Since the training algorithm has no prior knowledge about the training observations, the model can be transformed quickly according to the requirements of the user.

The machine-learning strategies also act as a tool to improve our knowledge of the underlying physics that controls the solar wind-magnetosphere coupling. On these grounds, a solution to find an alternative to the PC index, using different coupling functions, is also considered as a goal of the present work. The analysis shows that KanLee field $\left(E_{\mathrm{KL}}\right)$ can be considered as the best 
replacement for the $\mathrm{PC}$ index in predicting the $\mathrm{AE}$ index. Even though a lot of effort has been taken using multiple techniques, the problem of accuracy concerned with the prediction of the AE indices still looms large over the geomagnetic activity forecasting as much work has to be done to improve the statistical performance that can be expected from a long-term forecast. The major cause for this poor accuracy is related with the rapid changes in the $\mathrm{AE}$ which is in turn associated with variations in solar wind conditions and magnetospheric responses. The magnetic signatures associated with the auroral current system are related both to enhanced convection during the southward turning of the IMF and also to current wedge formations during the substorm triggering in the near midnight sector.

It is indeed doubtless that whatever be the forecasting strategy used, predicting long term (many steps in advance) is more difficult than predicting short term as the missing information will also increase with the unknown future time steps. The network time (which extends from a few seconds to several minutes or hours according to the length of the training data and prediction steps) necessary for training and testing the forecasting algorithm is also posing a serious limitation in using various time-series forecasting methods to real-world problems. Another problem is the proper choice of certain tuning parameters for the successful prediction of future values which differ from one system to another. Hence, the user always seeks a model that could learn faster, with minimum tuning parameters, and at the same time, one having better accuracy in predicting future values even when the output variable is trained with multiple inputs.

\section{Acknowledgements}

The authors are thankful to the World Data Center for Geomagnetism, Kyoto, Japan for providing AE, SYM and ASY data (http://wdc.kugi. kyoto-u.ac.jp/) and to USGS for providing 1-min Dst index data (http://geomag.usgs.gov/). The authors thank the World Data Center for Geomagnetism, Copenhagen (maintained by Technical University of Denmark) for providing 1-min PC index (ftp://ftp.space.dtu.dk/WDC/). The authors thank the OMNIWeb for providing the solar wind data (http://omniweb.gsfc.nasa.gov/). The authors thank C C Chang and C J Lin for providing the LibSVM package (https://www. csie.ntu.edu.tw/ cjlin/libsvm/), A Liaw and $\mathrm{M}$ Wiener for the Random Forest package (http:// cran.r-project.org/web/packages/randomForest/), G B Huang for providing the ELM and OS-ELM codes (http://www3.ntu.edu.sg/home/egbhuang/ elm_codes.html) and D Atabay for the pyrenn package (https://pyrenn.readthedocs.io). One of the authors, SG, extends his regards to Dr Madhu S Nair, assistant professor, Department of Computer Science, University of Kerala and to Dr K Satheesh Kumar, associate professor, Department of Future Studies, University of Kerala for their help and support. He also acknowledges a junior research fellowship (Ac.EVI(4)/17465/JRF/2017) from the University of Kerala, Trivandrum.

\section{References}

Akasofu S I 1975 The roles of the north-south component of the interplanetary magnetic field on large-scale auroral dynamics observed by the DMSP satellite; Planet. Space Sci. 23 1349-1354. https://doi.org/10.1016/ 0032-0633(75)90030-6.

Akasofu S I 2004 Several 'controversial' issues on substorms; Space Sci. Rev. 113 1-40.

Alexeev I I, Belenkaya E S and Kalegaev V V et al. 1996 Magnetic storms and magnetotail currents; J. Geophys. Res. Phys. 101 7737-7747, https://doi.org/10. 1029/95ja03509.

Amariutei O A and Ganushkina N Y 2012 On the prediction of the auroral westward electrojet index; Ann. Geophys. 30 841-847, https://doi.org/10.5194/angeo-30-841-2012.

Amata E, Consolini G, Pallocchia G and Marcucci M 2009 ANN forecast of hourly averaged AE index based on L1 IMF and plasma measurements; Acta Geophys. 57 185196, https://doi.org/10.2478/s11600-008-0083-1.

Anastasiadis A D, Magoulas G D and Vrahatis M N 2005 New globally convergent training scheme based on the resilient propagation algorithm; Neurocomputing 64 253270, https://doi.org/10.1016/j.neucom.2004.11.016.

Anderson J A, Rosenfeld E and Pellionisz A 1988 Neurocomputing; MIT Press, Cambridge, MA.

Baker D N, McPherron R L, Cayton T E and Klebesadel R W 1990 Linear prediction filter analysis of relativistic electron properties at 6.6 RE; J. Geophys. Res. 95 15,13315,140, https://doi.org/10.1029/JA095iA09p15133.

Bargatze L F, Baker D N, Hones E W and Mcpherron R L 1985 Magnetospheric impulse response for many levels of geomagnetic activity; J. Geophys. Res. 90 6387-6394, https://doi.org/10.1029/JA090iA07p06387.

Boberg F, Wintoft P and Lundstedt H 2000 Real time Kp predictions from solar wind data using neural networks; Phys. Chem. Earth 25 275-280, https://doi.org/10.1016/ S1464-1917(00)00016-7.

Breiman L 1996 Bagging predictors; Mach. Learn. 24 123140, https://doi.org/10.1007/BF00058655.

Breiman L 2001 Random forests; Mach. Learn. 45 5-32, https://doi.org/10.1023/A:1010933404324. 
Breiman L, Friedman J H, Olshen R A and Stone C J 1984 Classification and regression trees; Chapman \& Hall/CRC, Boca Raton.

Cai L, Ma S Y and Zhou Y L 2010 Prediction of SYM$\mathrm{H}$ index during large storms by NARX neural network from IMF and solar wind data; Ann. Geophys. 28 381393, https://doi.org/10.5194/angeo-28-381-2010.

Clauer C R and Kamide Y 1985 DP 1 and DP 2 current systems for the March 22, 1979 substorms; J. Geophys. Res. 90 1343, https://doi.org/10.1029/JA090iA02p01343.

Clauer C R, McPherron R L, Searls C and Kivelson M G 1981 Solar wind control of auroral zone geomagnetic activity; Geophys. Res. Lett. 8 915-918, https://doi.org/10.1029/ GL008i008p00915.

Dietterich T G 2000 Experimental comparison of three methods for constructing ensembles of decision trees: Bagging, boosting, and randomization; Mach. Learn. 40 139-157, https://doi.org/10.1023/A:1007607513941.

Drucker H, Burges C J C and Kaufman L et al. 1997 Support vector regression machines; Adv. Neural. Inf. Process. Syst. 9 155-161.

Friedrich E, Rostoker G, Connors M G and McPherron R L 1999 Influence of the substorm current wedge on the Dst index; J. Geophys. Res. Phys. 104 4567-4575, https:// doi.org/10.1029/1998ja900096.

Gannon J L and Love J J 2011 USGS 1-min dst index; J. Atmos. Sol.-Terr. Phys. 73 323-334, https://doi.org/10. 1016/j.jastp.2010.02.013.

Gavrishchaka V V and Ganguli S B 2001 Support vector machine as an efficient tool for high-dimensional data processing: Application to substorm forecasting; J. Geophys. Res. Sp. Phys. 106 29,911-29,914, https://doi.org/ 10.1029/2001JA900118.

Geurts P, Ernst D and Wehenkel L 2006 Extremely randomized trees; Mach. Learn. 63 3-42, https://doi.org/10. 1007/s10994-006-6226-1.

Gleisner H and Lundstedt H 1997 Response of the auroral electrojets to the solar wind modeled with neural networks; J. Geophys. Res. 102 14269, https://doi.org/10. 1029/96JA03068.

Gleisner H and Lundstedt H 1999 Ring current influence on auroral electrojet predictions; Ann. Geophys. 17 1268.

Gleisner H and Lundstedt H 2001 Auroral electrojet predictions with dynamic neural networks; J. Geophys. Res. 106 24,541-24,549, https://doi.org/10.1029/2001ja900046.

Gopinath S, Suji K J and Prince P R 2015 Information measures based analysis of complex solar windmagnetosphere interaction dynamics during geomagnetic storms; Indian J. Phys. 89(8) 759-772, https://doi.org/ $10.1007 / \mathrm{s} 12648-015-0655-2$.

Hammerstrom D 1993 Working with neural networks; IEEE Spectr. 30 46-53, https://doi.org/10.1109/6.222230.

Hernandez J V, Tajima T and Horton W 1993 Neural net forecasting for geomagnetic activity; Geophys. Res. Lett. 20 2707-2710, https://doi.org/10.1029/93GL02848.

Huang G-B, Zhu Q and Siew C et al. 2006 Extreme learning machine: Theory and applications; Neurocomputing $\mathbf{7 0}$ 489-501, https://doi.org/10.1016/j.neucom.2005.12.126.

Huang G B, Wang D H and Lan Y 2011 Extreme learning machines: A survey; Int. J. Mach. Learn. Cybern. 2 107122, https://doi.org/10.1007/s13042-011-0019-y.
Huang G, Huang G B, Song S and You K 2015 Trends in extreme learning machines: A review; Neural Netw. 61 $32-48$.

Iyemori T, Maeda H and Kamei T 1979 Impulse response of geomagnetic indices to interplanetary magnetic field; J. Geomagn. Geoelectr. 31 1-9.

Jia X, Wang R, Liu J and Powers D M W 2016 A semisupervised online sequential extreme learning machine method; Neurocomputing 174 168-178, https://doi.org/ 10.1016/j.neucom.2015.04.102.

King J H and Papitashvili N E 2005 Solar wind spatial scales in and comparisons of hourly wind and ACE plasma and magnetic field data; J. Geophys. Res. 110 A02104, https://doi.org/10.1029/2004JA010649.

Klimas A J, Baker D N and Roberts D A et al. 1992 A nonlinear dynamical analogue model of geomagnetic activity; J. Geophys. Res. 97 12253, https://doi.org/10. 1029/92JA00794.

Klimas A J, Vassiliadis D and Baker D N 1997 Data-derived analogues of the magnetospheric dynamics; J. Geophys. Res. 10226993.

Kugblenu S, Taguchi S and Okuzawa T 1999 Prediction of the geomagnetic storm associated Dst index using an artificial neural network algorithm; Earth Planet. Space $\mathbf{5 1}$ 307-313.

Lazzús J A, López-Caraballo C H, Rojas P, Salfate I, Rivera M and Palma-Chilla L 2016 Forecasting of Dst index from auroral electrojet indices using time-delay neural network + particle swarm optimization; J. Phys. Conf. Ser. $\mathbf{7 2 0} 012001$.

Liang N-Y, Huang G-B, Saratchandran P and Sundararajan N 2006 A fast and accurate online sequential learning algorithm for feedforward networks; IEEE Trans. Neural Netw. 17 1411-1423, https://doi.org/10.1109/TNN.2006. 880583.

Liemohn M W, Kozyra J U and Thomsen M F et al. 2001 Dominant role of the asymmetric ring current in producing the stormtime Dst; J. Geophys. Res. Sp. Phys. 106 10,883-10,904, https://doi.org/10.1029/2000JA000326.

Liu H L H 2010 On the Levenberg-Marquardt training method for feed-forward neural networks; In: Natural computation (ICNC), 2010 sixth international conference 1, https://doi.org/10.1109/ICNC.2010.5583151.

Lundstedt H and Wintoft P 1994 Prediction of geomagnetic storms from solar wind data with the use of a neural network; Ann. Geophys. 12 19-24, https://doi.org/10.1007/ s00585-994-0019-2.

McPherron R L, Baker D N and Bargatze L F et al. 1988 IMF control of geomagnetic activity; Adv. Sp. Res. 8 71-86, https://doi.org/10.1016/0273-1177(88)90114-7.

Munsami V 2000 Determination of the effects of substorms on the storm-time ring current using neural networks; J. Geophys. Res. 105, https://doi.org/10.1029/ 2000ja000041.

Pallocchia G, Amata E and Consolini G et al. 2008 AE index forecast at different time scales through an ANN algorithm based on L1 IMF and plasma measurements; J. Atmos. Solar-Terr. Phys. 70 663-668, https://doi.org/ 10.1016/j.jastp.2007.08.038.

Pavlov Y L 1997 Random forests; In: Probabilistic methods in discrete mathematics, (eds) Kolchin V F, Kozlov V Y, Pavlov Y L and Prokhorov Y V, Proc. 4th International 
Petrozavodsk Conf. 1996, VSP Publishers, Utrecht, The Netherlands, pp. 11-18.

Price C P and Prichard D 1993 The non-linear response of the magnetosphere: 30 October 1978; Geophys. Res. Lett. 20 771-774, https://doi.org/10.1029/93GL00844.

Revallo M, Valach F, Hejda P and Bochníček J 2014 A neural network Dst index model driven by input time histories of the solar wind-magnetosphere interaction; J Atmos. Sol.-Terr. Phys. 110-111 9-14, https://doi.org/10.1016/ j.jastp.2014.01.011.

Riedmiller M 1994 Advanced supervised learning in multilayer perceptrons - From backpropagation to adaptive learning algorithms; Comput. Stand. Interfaces 16265 278, https://doi.org/10.1016/0920-5489(94)90017-5.

Riedmiller M and Braun H 1993 A direct adaptive method for faster backpropagation learning: The RPROP algorithm; In: IEEE international conference on neural networks - Conference proceedings, pp. 586-591.

Rostoker G, Akasofu S I, Baumjohann W, Kamide Y and McPherron R L 1987 The roles of direct input of energy from the solar wind and unloading of stored magnetotail energy in driving magnetospheric substorms; Space. Sci. Rev. 4693.

Rostoker G, Akasofu S I and Baumjohann W et al. 1988 The roles of direct input of energy from the solar wind and unloading of stored magnetotail energy in driving magnetospheric substorms; Space. Sci. Rev. 4 93-111, https:// doi.org/10.1007/BF00173876.

Rumelhart D E and McClelland J L 1986 Parallel distributed processing; MIT Press, Cambridge, MA.

Stepanova M V and Pérez P 2000 Autoprediction of Dst index using neural network techniques and relationship to the auroral geomagnetic indices; Geofís. Int. 39 143-146.
Takalo J and Timonen J 1997 Neural network prediction of AE data; Geophys. Res. Lett. 24 2403-2406.

Takalo J and Timonen J 1999 Neural network prediction of the AE index from the PC index; Phys. Chem. Earth, C, Sol. Terr. Planet Sci. 24 89-92, https://doi.org/10.1016/ S1464-1917(98)00013-0.

Troshichev O and Janzhura A 2012 Space weather monitoring by ground-based means: $P C$ index; SpringerVerlag, Berlin, Heidelberg, 288p. https://doi.org/10. 1007/978-3-642-16803-1.

Unnikrishnan K 2014 Prediction of horizontal component of earth's magnetic field over Indian sector using neural network model; J. Atmos. Sol.-Terr. Phys. 121 206-220, https://doi.org/10.1016/j.jastp.2014.06.014.

Vapnik V 1998 The support vector method of function estimation; In: Nonlinear modeling: Advanced Black-Box Techniques (eds) Suykens J A K and Vandewalle J, Kluwer Academic Publishers, Boston, pp. 55-85.

Vapnik V and Chapelle O 2000 Bounds on error expectation for support vector machines; Neural. Comput. 12 20132036, https://doi.org/10.1162/089976600300015042.

Vassiliadis D, Klimas A J, Baker D N and Roberts D A 1995 A description of the solar wind-magnetosphere coupling based on nonlinear filters; J. Geophys. Res. 100 3495, https://doi.org/10.1029/94ja02725, ISSN 0148-0227.

Weigel R S, Horton W, Tajima T and Detman T 1999 Forecasting auroral electrojet activity from solar wind input with neural networks; Geophys. Res. Lett. 26 1353-1356, https://doi.org/10.1029/1999gl900280.

Weygand J M and McPherron R L 2006 Dependence of ring current asymmetry on storm phase; J. Geophys. Res. Sp. Phys. 111 A11221 2156-2202, https://doi.org/10.1029/ 2006JA011808. 